\title{
TYPOLOGIE ROMANE ET POSITION DES PRONOMS PERSONNELS CLITIQUES EN ROUMAIN
}

0 . Plusieurs auteurs ont décrit de façon détaillée les problèmes liés à la position des pronoms objets clitiques du roumain contemporain. Nous nous référons notamment aux inventaires systématiques de A. Lombard (1972), de M. Manoliu (1968) et de M. Iliescu (1975), à l'approche typologique romane de A. Niculescu (1973), aux études contrastives de R. Marinescu (1974 e.a.), et surtout à la dissertation de J. Bredemeier (1976), qui s'inspire de la thèse de D. Perlmutter, selon lequel les clitiques romans obéissent à des contraintes de surface (Surface Structure Constraints).

C'est une double préoccupation qui nous incite à reprendre en considération la problématique de la position des clitiques. Nous voudrions examiner la situation du roumain parmi les langues romanes d'une part, afin de tenter d'expliquer, d'autre part, en recourant au principe des corrélations typologiques, quelques particularités roumaines à peine élucidées.

\section{Le parallélisme avec les autres langues romanes est frappant:}

(a) les pronoms personnels objets direct et indirect s'agglutinent, avec d'autres morphèmes, au lexème verbal, et forment ainsi avec celui-ci une seule unité accentuelle;

(b) comme tout morphème agglutiné, ils occupent une place fixe et se succèdent dans un ordre rigide;

(c) d'une manière générale, la proclise est dominante, l'enclise récessive;

(d) les clitiques préfèrent partout que l'objet indirect précède l'objet direct (OI $>\mathrm{OD})$ et que les personnes du discours précèdent la $3 \mathrm{e}$ personne $(\mathrm{p} 1 / \mathrm{p} 2>\mathrm{p} 3)$

Parmi les traits qui individualisent le roumain, nous retiendrons les faits suivants dont la corrélation cadre dans l'adéquation explicative de cet exposé:

(a) les clitiques présentent plus d'allomorphes que dans les autres langues romanes;

(b) le gérondif employé seul déclenche l'enclise des pronoms (văzîndu-mă)

(c) le seul clitique o (p.3 acc. fém. sg.) est enclitique dans certains environnements, notamment derrière le part. passé du passé composé (am văzut-o), du futur antérieur (voi fi văzut-o), du conditionnel passé (aş fi văzut-o), et derrière l'infinitif du futur marqué par oi, etc. du conditionnel (aş vedea-o). 
Il est clair que le roumain, comme toutes les langues romanes d'ailleurs, favorise l'antéposition des clitiques, en accord avec la tendance romane à la prédétermination. Mais il y a plus: en limitant l'enclise à quelques contextes spécifiques seulement de la forme non-finie du verbe (V), le roumain occupe une position intermédiaire entre le français, où la proclise est de rigueur ${ }^{1}$ et les autres langues romanes, où l'enclise s'observe avec tout le paradigme non-fini du V. Ce comportement est conforme à la typologie générale de la morpho-syntaxe verbale du roumain qui tend, plus que la majorité des langues romanes, vers la prédétermination des morphèmes grammaticaux au lexème verbal: non seulement les auxiliaires (Aux) du passé, du futur, du conditionnel, mais aussi les marques du subjonctif (să) et de l'infinitif (a) s'antéposent.

Comme le roumain se caractérise vraisemblablement en même temps par un principe séquentiel qui rapproche les morphèmes porteurs de l'information sur les personnes (les arguments de la proposition)², il s'est créé pour les clitiques personnels une double contrainte positionnelle, qui n'a guère été valorisée dans les descriptions antérieures.

En effet, d'un côté le clitique personnel s'antépose immédiatement à la forme verbale finie, avec laquelle la cohésion sera d'autant plus forte que cette forme est un Aux, porteur d'information grammaticale, non lexicale. On notera entre le clitique et l'Aux non seulement l'impossibilité d'intercaler des adverbes, mais également l'effacement des limites syllabiques, deux faits qui sont facultatifs - sous des conditions définies - devant le lexème verbal:

îl mai arată

* Îl mai am arătat îl arată / L-arată

* Îl am arătat / L-am arătat

Que l'on compare l'emploi lexical et l'emploi grammaticalisé de $a$ avea dans:

Îsi are familia la ţară

Şi-a văzut familia

Il résulte de la tendance à la prédétermination et du principe de cohésion entre les morphèmes de personne que de l'autre côté le clitique suivra forcément les marques verbales antéposées qui ne portent pas sur la personne (să-l văd). De cette

1 Dans notre analyse, nous faisons abstraction de l'impératif affirmatif dont l'enclise se manifeste dans toutes les langues romanes. Le puissant accent d'intensité qui frappe cette forme expressive, et la distingue ainsi de façon supra-segmentale des autres formes verbales empêche probablement les clitiques atones de s'antéposer, à moins qu'ils puissent s'appuyer sur un élément dont l'anticipation est inévitable.

2 Ce principe sous-tend peut-être aussi la généralisation d'un pronom clitique possesseur auprès du V dans une construction non-marquée, plutôt qu'un morphème de possession dans le syntagme nominal (ii spală mașina $/ \mathrm{v} /$ spală mașina lui). 
façon, le clitique roumain se situe, au niveau distributionnel, toujours devant la forme verbale accompagnée de l'information itérative sur la personne du sujet, et au cas échéant derrière les marques verbales qui n'informent pas sur la catégorie de la personne ${ }^{3}$.

Le principe de la cohésion des marques de la personne implique aussi qu'une marque non-personnelle postposée n'attire le clitique à sa droite que lorsque la forme finie est également postposée (văzutu-l-am), ou qu'il n'y a pas de forme finie. Ainsi l'enclise au gérondif (arătîndu-le-o) n'apparaît plus comme exceptionnelle, mais correspond au contraire à la double contrainte distributionnelle.

Inversement, la double contrainte implique que l'antéposition du clitique au $\mathrm{V}$ aura toujours lieu, dès qu'une forme verbale est précédée soit d'une forme finie sans marque interposée (il voi vedea /v/am să-l văd), soit d'une marque modale, comme c'est le cas de $a$ pour l'infinitif ( $a-l$ vedea) ou de să pour le subjonctif (să $t i-l$ fi arătat). On comprend en même temps la position des clitiques dans les variantes du futur avec $o$ să et am să, où le clitique se place, conformément à la double contrainte, entre la marque non-personnelle et la forme finie du complexe morphologique verbal.

L'existence de la double contrainte rend plus évident, enfin, le parallélisme avec le français et l'écart avec les autres langues romanes. Tout comme en français la présence quasi générale d'un clitique sujet à gauche, qui constitue avec le $\mathrm{V}$ un seul mot phonétique et est dominé ainsi par le noeud $\mathrm{V}$ plutôt que par le noeud $\mathrm{SV}^{4}$, expliquerait en partie la généralisation absolue de la proclise, le développement diachronique de morphèmes verbaux à gauche du lexème verbal favorise la proclise en roumain (tendence à l'homogénéité syntaxique), à tel point que l'enclise ne se limite plus qu'aux quelques formes non-finies postdéterminées.

2. C'est dans cette optique qu'il convient d'évaluer le comportement aberrant du pronom $o$ qui n'obéit ni au principe de la cohésion des morphèmes de personne, ni à la double contrainte distributionnele dans quelques environnements avec l'infinitif ou le participe passé, où $o$ est obligatoirement enclitique:

Am văzut-o

Ion ar cunoaşte-o

Aṣ. fi văzut-o

Oi cunoaşte-o

${ }^{3}$ Que l'on compare avec les descriptions de R. Marinescu (1973: 233), qui définit les clitiques roumains à gauche par zéro, en opposition avec le français.

4 Nous faisons allusion ici à l'analyse proposée par R. Kayne (1977: 86sv.). 
L'agrammaticalité de $o$ en proclise tient - on le sait - - au vocalisme initial de l'Aux. Dès que l'Aux commence par une consonne, l'interdiction est levée:

Ion o va cunoaşte ${ }^{5}$

Aș fi vrut s-o fi văzut

C'est que la contrainte qui bloque l'application des règles syntaxiques formulées ci-dessus, est de nature phonologique. Ce n'est pas neuf. J. Bredemeier (1976: 37-42) insistait déjà sur le fait que du point de vue phonotactique, $o$ se comporte dans certaines structures comme un Aux commençant par une voyelle; l'enclise de $o$ s'expliquerait ainsi par une collision homonymique avec l'Aux à initiale vocalique: "Die postverbale Stellung des $\mathrm{K} 1_{\mathrm{A}}[=$ clitique accusatif $] o$ hat sich in den Strukturen durchgesetzt und grammatikalisiert, in denen bei präverbaler Position die Gefahr einer Verwechslung oder einer Kollision mit einem Aux [ = Aux à initiale vocalique] bestand" (J. Bredemeier 1976: 41).

Pourtant cette thèse, très vraisemblable, ne s'applique pleinement qu'à un ensemble réduit des formes et combinaisons concernées. La réticence à l'égard de la proclise de $o$ tient à la fois à la physionomie exceptionnelle de ce morphème dans le paradigme morphologique des clitiques objets, et aux propriétés spécifiques de l'allomorphisme de ceux-ci. Malgré le grand nombre d'allomorphes, chaque clitique se caractérise par un noyau stable, qui fonctionne comme forme distinctive. Ce noyau formel est toujours / + consonantique/; en effet, l'allomorphisme affecte seulement les segments vocaliques ${ }^{6}$, qui n'interviennent que dans des buts phonotactiques, notamment quand une syllabe est nécessaire, p. ex. lorsque les limites morphémiques ne s'effacent pas (mă interesează, $\hat{\imath} l$ cunoaște ${ }^{7}$ ), ou en combinaison avec un autre clitique ( $m i-l d \vec{a}$, mi-i dă, $d \breve{a}-n i-l e$ !). Le tableau suivant montre la régularité remarquable du système morphologique:

\begin{tabular}{|c|c|c|c|c|c|}
\hline \multicolumn{2}{|c|}{ datif } & \multicolumn{4}{|c|}{ accusatif } \\
\hline sg & $\mathrm{pl}$ & \multicolumn{2}{|c|}{ sg } & \multicolumn{2}{|c|}{$\mathrm{pl}$} \\
\hline \multirow{3}{*}{$\begin{array}{l}1 m i \\
2 t i j \\
3 i d i\end{array}$} & \multirow{3}{*}{$\begin{array}{c}n(e) / n i \\
v / v i \\
l(e) / l i\end{array}$} & masc. & fém & masc. & fém \\
\hline & & \multicolumn{2}{|c|}{$\begin{array}{c}m \\
t(e)\end{array}$} & \multicolumn{2}{|c|}{$n(e)$} \\
\hline & & $l$ & $o$ & $i / i$ & $l(e)$ \\
\hline
\end{tabular}

${ }^{5}$ L'enclise de $o$ est facultative avec le futur marqué par voi... et relève du registre parlé et populaire (cf. A. Niculescu, 1973: 470). Ceci vaut également pour l'infinitif employé seul: a vedea-o, faptul de a fi văzut-o (ce dernier ex. d'après A. Lombard, 1972: 211).

6 A condition que l'on fasse abstraction de la structuration morphologique particulière de /p3 non

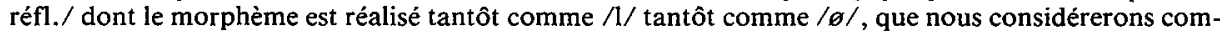
me les noyaux stables, à côté de l'inanalysable $o$ (cf. ci-après).

7 Comme les sonantes $(1, \mathrm{~m}, \mathrm{n}$ ) peuvent constituer le noyau d'une syllabe, le segment vocalique peut faire défaut dans le débit rapide: $l$ vad, l prind (cf. A. Lombard, 1972: 235). 
A première vue, deux formes seulement font exception au caractère $/+$ consonantique/ du noyau stable et distinctif du morphème. Ce sont $\hat{l} i$ à la fois $\mathrm{p} 3$ datif sg. et $\mathrm{p} 3$ acc. masc. pl., et $o, \mathrm{p} 3$ acc. fém. sg.

La première exception toutefois ne résiste pas à un examen approfondi. En fait, le noyau distinctif de ce clitique n'est pas $/ \underline{i} /$, mais $/ \phi /$, ensemble avec $/ 1 /$ allomorphe du morphème $/ \mathrm{p} 3$ non réfl/ ${ }^{8}$. /i/ est la marque tantôt du datif (cf. les autres clitiques au datif $m+i, t+i, s+i)$, tantôt du masc. pl. Cette analyse est confirmée (a) par le comportement morphonologique identique de la même marque auprès des autres clitiques du datif (îmi, ît $i$, ișsi), où i devient vocalique dans les mêmes conditions, (b) par l'existence des mêmes allomorphes / $\mathrm{i} /$ et /i/du masc. pl. dans d'autres environnements (p. ex. copii, codri), (c) par la présence parallèle de /e/ pour le fém. pl. dans le système des clitiques, (d) par le fait que le clitique $\hat{\imath} i$, qu'il se présente sous sa forme vocalique ou non, déclenche les mêmes modifications phonotactiques dans son environnement qu'un morphème consonantique, ce que nous attribuons à la présence du morphème $/ \phi /$ :

$\begin{array}{lll}\text { dîndu-1, dîndu-i-l } & \text { /v/ } & \text { dînd-o } \\ \text { arată-1, arată-i-l } & \text { /v/ } & \text { ascult-o } \\ \text { să-1 arătaţi, să i-l arătaţi } & \text { /v/ } & \text { s-o arătaţi }\end{array}$

Le caractère exceptionnel de $o$ dans le paradigme des clitiques saute aux yeux. C'est le seul clitique dont la forme distinctive est / + vocalique; - consonantique/; c'est aussi le seul clitique qui soit altérerait sa base formelle devant un Aux à initiale vocalique, soit ne formerait pas une seule syllabe avec l'Aux, à l'opposé des autres clitiques ( $(l-a$ dat, mi-a dat, même ne-a dat, te-oi vedea). Etant donné l'effacement des limites morphémiques devant l'Aux à initiale vocalique, et le témoignage de $S$. Pop (1948: 49) à propos de la prononciation luat-u-am de la langue parlée ${ }^{9}$, il est probable que le contact avec $a$ - ou $o$ - réduirait le clitique tantôt à zéro, tantôt à un segment /-vocalique/, à d'autant plus forte raison que, selon certains auteurs (cf. e.a. DOOM 1982: XI), la dévocalisation de oa serait plus forte en position initiale absolue que derrière consonne (oameni $[\mathrm{wa}] / \mathrm{v} /$ toată $\left.^{[\mathrm{oa}}\right]^{10}$. Ainsi ce serait le seul environnement où un clitique ne garderait pas intact son noyau formel distinctif, ce qui serait d'autant plus exceptionnel que le trait vocalique de $o$ l'emporte dans tous les autres contextes phoniques (cf. les exemples ci-dessus dînd-o, etc., de même que n-o vede, dati-o, v-o da, ne-o da, le-o da, prononcés /nöo/, /löo/ ou /nö/, /ö/ d'aprés I. Calota 1986: 12).

${ }^{8}$ Nous contestons ainsi l'analyse de R. Bley-Vroman (1977: 26), qui propose /e/ comme morphème $/ \mathrm{p} 3$, non réfl./, en considérant, sous l'inspiration de P. Postal, l'article défini et le pronom personnel comme des variantes de la même unité.

9 C qui contredirait la thèse de I. Calota (1986: 76) selon lequel une diphtongaison entre $o$ et $a$ serait exclue. Même sous cet angle, le comportement du clitique serait exceptionnel: il n'obéirait pas à l'effacement des limites morphémiques devant l'Aux vocalique.

10 Une différence entre /Qa/ et /uxa/ est contestée par I. Calota (1986: 18-26), en faveur de /ụa/. 
L'enclise de $o$ dans les constructions mentionnées s'avère ainsi être le résultat d'un conflit entre les lois prosodiques du roumain, où $a$ prime toujours sur $o$, moins ouvert, dans la synérèse, obligatoire devant l'Aux selon le principe de la cohésion des morphèmes personnels d'une part, et la stabilité du noyau distinctif vocalique du clitique /p3 acc. fém. $s g . /$ d'autre part, d'un conflit aussi entre l'élément dominant de la synérèse $(a>o)$ et l'information sémique dominante $(o>a$ Aux), entre les possibilités d'allomorphisme d'une forme à base / + consonantique/ et d'une forme à base / + vocalique, - consonantique/.

On déduit de cet ensemble complexe de principes en conflit, que $o$ abandonne l'enclise dès que sa base vocalique n'est plus guère menacée:

(a) devant un Aux à initiale consonantique (o voi vedea, s-o fi văzut);

(b) devant un lexème verbal, où la limite syllabique est facultative et où le clitique n'a pas de choix, selon sa définition distributionnelle (o ajută, o oprește come mă ajută, mă oprește);

(c) éventuellement, devant un Aux à initiale vocalique, à condition que $O$ soit précédé d'un autre clitique personnel, avec lequel il forme une syllabe à part entière"11.

En revanche, en cas d'inversion de l'Aux, les règles de placement clitique, telles qu'on vient de les formuler, interdisent à $o$ une autre position qu'entre le participe passé (postposition au morphème personnel): cf. les exemples archaïques du type văzutu-l-am.

Aussi $o$ est-il rare dans cette construction, relativement aux autres pronoms clitiques (cf. J. Bredemeier 1976: 41).

Enfin, les cas d'enclise facultative ne peuvent s'expliquer que par une tendance naturelle à la régularisation de la structure de surface " $A \mathrm{x}_{\mathrm{fut}}+\mathrm{V}$ non fini $+o^{\text {" et }}$ "Inf $+o$ " (a vedea-o, voi vedea-o, cf. note 5 ci-dessus)

3. Nous avons essayé de démontrer qu'une double contrainte distributionnelle (antéposition avec forte cohésion aux formes verbales finies; postposition aux morphèmes non-personnels) est responsable du comportement syntaxique des clitiques, auxquels seulement la forme $o$ fait exception, par ses propriétés formelles particulières. De cette façon, le roumain favorise la proclise, à tel point que l'enclise se limite aux formes non-finies postdéterminées (notamment le gérondif d'une part, et à des conditions d'ordre phonologique (valables pour o) d'autre part.

\footnotetext{
11 Cf les exemples archaïques de J. Bredemeier (1976: 38-39: cu urzici i-o am frecat) qui souligne le lien entre la proclise de $o$ et la présence d'un autre clitique.
} 


\section{BIBLIOGRAPHIE}

Bley-Vroman, R. (1977): "Rumanian Pronoun Morphology from a Generative Point of View", in: S. Alexandrescu (ed.): Transformational Grammar and the Rumanian Language. Lisse, De Ridder.

Bredemeier, J. (1979): Strukturbeschränkungen im Rumänischen. Studien zur Syntax der prä- und postverbalen Pronomina. Tübingen, Narr.

Calota, I. (1986): Contributii la fonetica şi dialectologia limbii române. Craiova, Scrisul Românesc.

DOOM: Dicționarul ortografic, ortoepic şi morfologic al limbii române, Bucarest, Ed. Academiei RSR, 1982.

Iliescu, M. (1975): "Pentru o sistematizare a predării pronumelui personal neaccentuat românesc", in: Limba Română, 24, 51-62.

Kayne, R. (1977): Syntaxe du français. Le cycle transformationnel. Paris, Seuil.

Lombard, A. (1972): Les pronoms personnels du roumain. Aperçu syntaxique, in: Studier i Modern Språkvetenskap 4, 190-249.

Manoliu, M. (1968): Sistematica substitutelor din româna contemporană standard, Bucarest, ed. Academiei R.S.R.

Marinescu, R. (1973): "La syntaxe des particules pronominales pré/postverbales en roumain et en français. Analyse contrastive des contraintes de surface", in: $\mathrm{Ca}$ hiers de linguistique théorique et appliquée, 10, 223-245.

Marinescu, R. (1974): “Comparaison morphosyntaxique des pronoms personnels en roumain et en français. Ahalyse distributionnelle des formes réduites ", in: $\mathrm{Bul}$ letin de la Société Roumaine de Linguistique Romane 10, 37-53.

Niculescu, A. (1973): "Pronominalizzazione clitica rumena e romanza. Approccio tipologico“, in: Revue Roumaine de Linguistique, 18, 467-476.

Pop, S. (1948): Grammaire roumaine, Berne, Francke.

Povzetek

ROMANSKA TIPOLOGIJA IN STAVA NENAGLAŠENIH OSEBNIH ZAIMKOV V ROMUNŠC̆INI

Nenaglašeni osebni zaimek kot objekt pozna $v$ romunšc̆ini enako skladnjo kot $v$ drugih romanskih jezikih: proklitična stava je skorajda pravilo, v soglasju s težnjo, da stoji determinant na prvem mestu, kar je nasploh značilnost morfologije romunskega glagola.

Imamo pa vendar dve izjemi: a) zapostavljanje za gerundijem; b) zapostavljanje zaimka 3.os.ed.ž.sp. $o$, še prav pösebej v sestavljenem pretéklem času.

Prvo odstopanje je mogoče razložiti z dvojno prisilo: stava spredaj je značilna pri osebnih glagolskih oblikah, pri čemer je kohezija močnejša, stava zadaj je značilnost neosebnih glagolskih oblik $\mathrm{v}$ več romanskih jezikih.

Odstopanje $v$ stavi zaimka $o$ je pogojeno $s$ fonotaktičnimi načeli. $Z$ ene strani je tako zagotovljena jasnost: ob trčenju samoglasnikov $o$ in $a$ zmeraj prevlada $a$ in potemtakem o am văzut ne bi bilo razumljivo, am văzut-o 'videl sem jo' pa je. Z druge strani pa gre za'priobčevalno strategijo, saj zapostavljeni zaimek $\boldsymbol{o}$ posreduje dominantno informacijo, pomembno za ves sestavljeni glagol. 